\title{
Research Article \\ Surfaces of Constant Curvature in the Pseudo-Galilean Space
}

\author{
Željka Milin Šipuš ${ }^{\mathbf{1}}$ and Blaženka Divjak ${ }^{2}$ \\ ${ }^{1}$ Department of Mathematics, Faculty of Science, University of Zagreb, Bijenička Cesta 30, \\ 10000 Zagreb, Croatia \\ ${ }^{2}$ Faculty of Organization and Informatics, University of Zagreb, Pavlinska 2, 42000 Varaždin, Croatia
}

Correspondence should be addressed to Željka Milin Šipuš, milin@math.hr

Received 16 May 2012; Accepted 1 July 2012

Academic Editor: Ram U. Verma

Copyright (c) 2012 Ž. Milin Šipuš and B. Divjak. This is an open access article distributed under the Creative Commons Attribution License, which permits unrestricted use, distribution, and reproduction in any medium, provided the original work is properly cited.

We develop the local theory of surfaces immersed in the pseudo-Galilean space, a special type of Cayley-Klein spaces. We define principal, Gaussian, and mean curvatures. By this, the general setting for study of surfaces of constant curvature in the pseudo-Galilean space is provided. We describe surfaces of revolution of constant curvature. We introduce special local coordinates for surfaces of constant curvature, so-called the Tchebyshev coordinates, and show that the angle between parametric curves satisfies the Klein-Gordon partial differential equation. We determine the Tchebyshev coordinates for surfaces of revolution and construct a surface with constant curvature from a particular solution of the Klein-Gordon equation.

\section{Introduction}

Study of differential geometry of curves and surfaces in Euclidean, as well as in other nonEuclidean ambient spaces, has a long history. Classical context of the Euclidean space is a source of results which could be transferred to some other geometries. One way of defining new geometries is through Cayley-Klein spaces. They are defined as projective spaces $p_{n}(\mathbf{R})$ with an absolute figure, a subset of $p_{n}(\mathbf{R})$ consisting of a sequence of quadrics and planes [1]. Projectivities of the projective space $p_{n}(\mathbf{R})$ which leave invariant the absolute figure define the subgroup of projectivities called the group of motions of a Cayley-Klein space. By means of the absolute figure, metric relations are also defined and they are invariant under the group of motions.

In three-dimensional projective space $p_{3}(\mathbf{R})$ various types of Cayley-Klein spaces can be defined, such as elliptic and hyperbolic space, Euclidean and pseudo-Euclidean 
(Minkowski) space, simple and double isotropic space, Galilean and pseudo-Galilean space, and quasielliptic and quasihyperbolic space. General theory of differential geometry of curves and surfaces in Cayley-Klein spaces can be found in [1]. Foundations of these areas in the pseudo-Galilean space were established in [2], as well as in the papers [3-7]. Geometry of the Galilean space $G_{3}$ was studied in [8-11]. The four-dimensional Galilean space appears in connection with classical Newtonian mechanics, where first coordinate describes time and other three coordinates are space coordinates.

The main interest of this paper is to develop the local theory of surfaces in the pseudoGalilean space and to study surfaces of constant curvatures. As in the Minkowski space [12], two classes of surfaces are introduced, spacelike and timelike surfaces, and for them the Gaussian curvature is defined. The obtained results are compared to the well-known results in the Euclidean and Minkowski geometry. The results can easily be transferred to the Galilean space. Furthermore, we define the Tchebyshev coordinates on a surface and show that the asymptotic lines form the Tchebyshev net if and only if this surface is a spacelike surface of constant negative curvature or timelike surface of constant positive curvature. We study the angle between the Tchebyshev curves on a surface of constant curvature and show that the angle satisfies the Klein-Gordon partial differential equation. In this respect, the Klein-Gordon equation plays the analogous role in the pseudo-Galilean space as the sine-Gordon equation in Euclidean space. We construct a surface with constant Gaussian curvature from a known solution of a Klein-Gordon equation. Similar problem is treated in [13] for the Galilean space and in wider context in [14] by means of Cartan frames.

\section{Preliminaries}

The absolute figure of the pseudo-Galilean space is the ordered triple $\{\omega, f, I\}$, where $\omega$ is the ideal (absolute) plane in the real three-dimensional projective space $p_{3}(\mathbf{R}), f$ the line (absolute line) in $\omega$, and $I$ the fixed hyperbolic involution of points of $f$. Homogeneous coordinates in $G_{3}^{1}$ are introduced in such a way that the absolute plane $\omega$ is given by $x_{0}=0$, the absolute line $f$ by $x_{0}=x_{1}=0$, and the hyperbolic involution by $\left(0: 0: x_{2}: x_{3}\right) \mapsto(0:$ $0: x_{3}: x_{2}$ ). The last condition is equivalent to the requirement that the conic $x_{2}^{2}-x_{3}^{2}=0$ is the absolute conic. Metric relations are introduced with respect to the absolute figure. In affine coordinates defined by $\left(x_{0}: x_{1}: x_{2}: x_{3}\right)=(1: x: y: z)$, distance between points $P_{i}=\left(x_{i}, y_{i}, z_{i}\right), i=1,2$, is defined by

$$
d\left(P_{1}, P_{2}\right)= \begin{cases}\left|x_{2}-x_{1}\right|, & \text { if } x_{1} \neq x_{2} \\ \sqrt{\left|\left(y_{2}-y_{1}\right)^{2}-\left(z_{2}-z_{1}\right)^{2}\right|,} & \text { if } x_{1}=x_{2} .\end{cases}
$$

The group of motions of $G_{3}^{1}$ is a six-parameter group given (in affine coordinates) by

$$
\begin{aligned}
& \bar{x}=a+x, \\
& \bar{y}=b+c x+y \cosh \varphi+z \sinh \varphi, \\
& \bar{z}=d+e x+y \sinh \varphi+z \cosh \varphi .
\end{aligned}
$$

It leaves invariant the absolute figure as well as the pseudo-Galilean distance (2.1) of points. 
In the pseudo-Galilean space, a vector is called isotropic if it is of the form $(0, y, z)$. Among these vectors, there are also vectors with supplementary norm $\sqrt{\left|y^{2}-z^{2}\right|}$ equal to zero; they are called lightlike vectors. Isotropic vectors satisfying $y^{2}-z^{2}>0$ are said to be spacelike vectors and vectors satisfying $y^{2}-z^{2}<0$ timelike vectors. A plane of the form $x=$ const. is called a pseudo-Euclidean plane (since its induced geometry is pseudo-Euclidean, i.e., Minkowski plane geometry), otherwise it is called isotropic (since its induced geometry is isotropic, i.e., Galilean plane geometry). In the pseudo-Euclidean plane distance between points $P_{i}, i=1,2$, given by their affine coordinates $P_{i}=\left(x_{i}, y_{i}\right)$ is defined by

$$
d\left(P_{1}, P_{2}\right)=\sqrt{\left|\left(x_{2}-x_{1}\right)^{2}-\left(y_{2}-y_{1}\right)^{2}\right|},
$$

while in the isotropic plane

$$
d\left(P_{1}, P_{2}\right)= \begin{cases}\left|x_{2}-x_{1}\right|, & \text { if } x_{1} \neq x_{2} \\ \left|y_{2}-y_{1}\right|, & \text { if } x_{1}=x_{2}\end{cases}
$$

The pseudo-Galilean space $G_{3}^{1}$ can be also regarded as a Cayley-Klein space equipped with the projective metric of signature $(0,0,+,-)$, as explained in [15]. According to the description of the Cayley-Klein spaces in [1], it is denoted by $P_{11 \mid 001}^{3}$ and also called the Galilean space of index 1.

\section{The Gaussian Curvature of Surfaces in $G_{3}^{1}$}

We will treat a $C^{r}$-surface, $r \geq 2$, as a subset $\Phi \subset G_{3}^{1}$ for which there exists an open subset $D$ of $\mathbf{R}^{2}$ and $C^{r}$-mapping $\mathbf{x}: D \rightarrow G_{3}^{1}$ satisfying $\Phi=\mathbf{x}(D)$. A $C^{r}$-surface $\Phi \subset G_{3}^{1}$ is called regular if $\mathbf{x}$ is an immersion and simple if $\mathbf{x}$ is an embedding. It is admissible if it does not have pseudoEuclidean tangent planes. Let us denote $\mathbf{x}=\mathbf{x}\left(x\left(u_{1}, u_{2}\right), y\left(u_{1}, u_{2}\right), z\left(u_{1}, u_{2}\right)\right), x_{, i}=\partial x / \partial u_{i}$, $y_{, i}=\partial y / \partial u_{i}, z_{, i}=\partial z / \partial u_{i}, i=1,2$. Then a surface is admissible if and only if $x, i \neq 0$, for some $i=1,2$. If we assume

$$
\left|\begin{array}{ll}
x, 1 & y_{1} \\
x_{, 2} & y_{, 2}
\end{array}\right| \neq 0
$$

then such a surface is admissible and can be locally expressed in the form

$$
z=z(x, y)
$$

Let $\Phi \subset G_{3}^{1}$ be a regular admissible surface. We define a side tangential vector by

$$
\sigma=\frac{1}{W}\left(x_{, 1} \mathbf{x}_{, 2}-x_{, 2} \mathbf{x}_{, 1}\right)
$$


The vector $\sigma$ is a vector in a tangent plane and we assume that it is not an isotropic lightlike vector, but a unit isotropic spacelike or timelike vector. The function $W, W>0$, defined by

$$
W=\sqrt{\left|\left(x_{, 1} y_{, 2}-x_{, 2} y_{, 1}\right)^{2}-\left(x_{, 1} z_{, 2}-x_{, 2} z_{, 1}\right)^{2}\right|}
$$

is equal to the pseudo-Galilean norm of the isotropic vector $x_{, 1} \mathbf{x}_{, 2}-x_{, 2} \mathbf{x}_{, 1}$. In particular, in the parametrization (3.2) we have

$$
\sigma=\frac{1}{W}(0,1, z, y), \quad W=\sqrt{\left|1-z_{, y}^{2}\right|}
$$

In the following, we will not consider surfaces with $W=0$, that is, surfaces having lightlike side tangential vectors (lightlike surfaces).

In a tangent plane in a point $P_{0}$ of a regular admissible surface, there is a unique isotropic direction defined by the condition $x_{, 1} \mathrm{~d} u_{1}+x_{, 2} \mathrm{~d} u_{2}=0$. This isotropic line in a tangent plane in a point $P_{0}$ of the surface meets the absolute line $f$ in a point $S$. If we denote by $S^{\perp}$ a point on $f$ obtained from $S$ by the hyperbolic involution $I$, then a line connecting $P_{0}$ and $S^{\perp}$ is perpendicular to the tangent plane. Therefore a unit surface normal field is defined by

$$
\mathbf{N}=\frac{1}{W}\left(0, x_{, 1} z, 2-x_{, 2} z, 1, x_{, 1} y_{, 2}-x_{, 2} y_{1}\right)
$$

We introduce a pseudo-Galilean cross-product in the following way:

$$
\mathbf{a} \times_{G} \mathbf{b}=\left|\begin{array}{ccc}
\mathbf{0} & -\mathbf{e}_{2} & \mathbf{e}_{3} \\
a_{1} & a_{2} & a_{3} \\
b_{1} & b_{2} & b_{3}
\end{array}\right|,
$$

where $\mathbf{e}_{2}=(0,1,0), \mathbf{e}_{3}=(0,0,1), \mathbf{e}_{2}$ is a unit spacelike, and $\mathbf{e}_{3}$ is a unit timelike vector, $\mathbf{a}=$ $\left(a_{1}, a_{2}, a_{3}\right), \mathbf{b}=\left(b_{1}, b_{2}, b_{3}\right)$. Now we can write

$$
\mathbf{N}=\frac{1}{W} \mathbf{x}_{1} \times_{G} \mathbf{x}_{2}
$$

which for (3.2) turns to $\mathbf{N}=(1 / W)\left(0, z_{y}, 1\right)$. Furthermore, we can notice that the pseudoGalilean cross product can be defined by means of the pseudo-Galilean scalar product so that $\mathbf{a} \times_{G} \mathbf{b}$ is the isotropic vector defined by the relation

$$
\left(\mathbf{a} \times_{G} \mathbf{b}\right) \cdot \mathbf{c}=-\operatorname{det}(\mathbf{a}, \mathbf{b}, \tilde{\mathbf{c}}),
$$

for any vector c. By above a vector, the projection of a vector on the pseudo-Euclidean $y z-$ plane is denoted and by - the pseudo-Euclidean scalar product in the same plane, $\left(y_{1}, y_{2}\right)$. $\left(z_{1}, z_{2}\right)=y_{1} z_{1}-y_{2} z_{2}$.

Obviously the following proposition holds. 
Proposition 3.1. The side tangential vector $\sigma$ and the normal vector $\mathbf{N}$ are unit isotropic vectors that satisfy

$$
\sigma \cdot \mathbf{N}=0, \quad \mathbf{N}^{2}:=\epsilon= \pm 1, \quad \sigma^{2}=-\epsilon .
$$

Since the normal vector field satisfies $\mathbf{N} \cdot \mathbf{N}=\epsilon= \pm 1$, we distinguish two basic types of admissible surfaces: spacelike surfaces having timelike surface normals $(\epsilon=-1)$ and timelike surfaces having spacelike normals $(\epsilon=1)$. A surface is spacelike if $\left(x_{, 1} \mathbf{x}_{, 2}-x_{, 2} \mathbf{x}_{, 1}\right)^{2}=\left(x_{, 1} y_{, 2}-\right.$ $\left.x_{, 2} y_{1}\right)^{2}-\left(x_{, 1} z, 2-x_{, 2} z, 1\right)^{2}>0$ in all of its points, timelike otherwise. In the parametrization (3.2) a surface is spacelike if $1-z_{, y}^{2}>0$.

The first fundamental form of a surface is induced from the metric of the ambient space $G_{3}^{1}$

$$
\mathrm{d} s^{2}=\left(x_{, 1} \mathrm{~d} u_{1}+x_{, 2} \mathrm{~d} u_{2}\right)^{2}+\delta\left(\widetilde{\mathbf{x}}_{, 1} \mathrm{~d} u_{1}+\widetilde{\mathbf{x}}_{, 2} \mathrm{~d} u_{2}\right)^{2},
$$

where

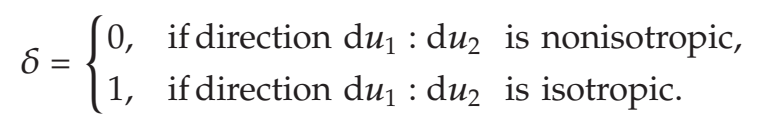

We introduce the fundamental coefficients

$$
g_{i}=x_{, i}, \quad h_{i j}=\tilde{\mathbf{x}}_{, i} \cdot \tilde{\mathbf{x}}_{, j}, \quad i, j=1,2
$$

by which the first fundamental form can be written as

$$
\mathrm{d} s^{2}=\left(g_{1} \mathrm{~d} u_{1}+g_{2} \mathrm{~d} u_{2}\right)^{2}+\delta\left(h_{11} \mathrm{~d} u_{1}^{2}+2 h_{12} \mathrm{~d} u_{1} \mathrm{~d} u_{2}+h_{22} \mathrm{~d} u_{2}^{2}\right)
$$

Notice again that the indices $i$ or $i j$ on variables denote different symbols, whereas indices, $i$ or, $i, j$ denote the partial derivatives with respect to the $i$ th and $j$ th parameter. Furthermore $h_{i j}=h_{j i}, g_{i, j}=g_{j, i}, i=1,2$.

Now the function $W$ has the form

$$
\begin{aligned}
W^{2} & =-\epsilon\left(g_{1} \mathbf{x}_{, 2}-g_{2} \mathbf{x}_{, 1}\right)^{2} \\
& =-\epsilon\left(g_{1}^{2} h_{22}-2 g_{1} g_{2} h_{12}+g_{2}^{2} h_{11}\right)>0
\end{aligned}
$$

and the surface is spacelike if $g_{1}^{2} h_{22}-2 g_{1} g_{2} h_{12}+g_{2}^{2} h_{11}>0$, timelike otherwise. We can notice that if a surface is spacelike, both parts of the first fundamental form, $\mathrm{d} s_{1}^{2}=\left(g_{1} \mathrm{~d} u_{1}+g_{2} \mathrm{~d} u_{2}\right)^{2}$ and

$$
\mathrm{d} s_{2}^{2}=h_{11} \mathrm{~d} u_{1}^{2}+2 h_{12} \mathrm{~d} u_{1} \mathrm{~d} u_{2}+h_{22} \mathrm{~d} u_{2}^{2}=-\epsilon \frac{W^{2}}{g_{1}^{2}} \mathrm{~d} u_{2}^{2}, \quad g_{1} \neq 0
$$




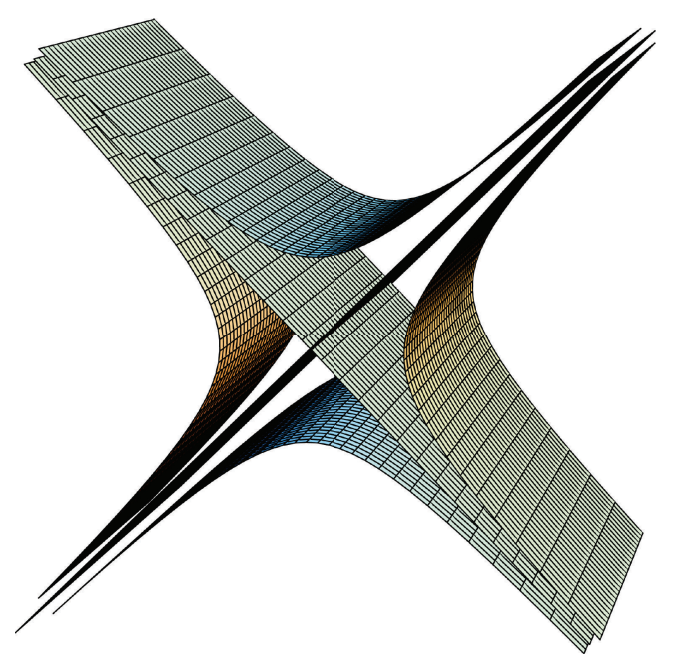

Figure 1: Lightlike planes, a spacelike and a timelike hyperbolic sphere.

are positive definite, while for the timelike surfaces, the form $\mathrm{d} s_{1}^{2}$ is positive definite whereas $\mathrm{d} s_{2}^{2}$ is negative definite. We have assumed here, without loss of generality, $g_{1} \neq 0$. In the latter case this means that the matrix of the first fundamental form

$$
\left(\begin{array}{cc}
\mathrm{d} s_{1}^{2} & 0 \\
0 & \mathrm{~d} s_{2}^{2}
\end{array}\right)
$$

is indefinite, analogously to the timelike surfaces in the Minkowski space $\mathbf{R}_{1}^{3}$ [12].

In particular, for the parametrization (3.2) we have

$$
\mathrm{d} s^{2}=\mathrm{d} x^{2}+\delta\left(1-z_{, y}^{2}\right) \mathrm{d} y^{2}
$$

since $\mathrm{d} x=0$ when $\delta=1$.

Example 3.2. Hyperbolic cylinders $z^{2}-y^{2}=R^{2}\left(y^{2}-z^{2}=R^{2}\right)$ are surfaces which are everywhere spacelike (timelike). They are spheres of the space $G_{3}^{1}$, called hyperbolic spheres. Planes $y^{2}-z^{2}=0$ are everywhere lightlike surfaces, see Figure 1 .

The Gaussian curvature of a surface is defined by means of the coefficients of the second fundamental form $L_{i j}, i, j=1,2$, which are the normal components of $\mathbf{x}_{i, j}, i=1,2$, respectively. If we put

$$
\mathbf{x}_{, i, j}=\sum_{k=1}^{2} \Gamma_{i j}^{k} \mathbf{x}_{, k}+L_{i j} \mathbf{N}
$$

then the following proposition follows. 
Proposition 3.3. One has

$$
L_{i j}=\epsilon \frac{1}{g_{1}}\left(g_{1} \tilde{\mathbf{x}}_{i, j}-g_{i, j} \tilde{\mathbf{x}}_{, 1}\right) \cdot \mathbf{N}=\epsilon \frac{1}{g_{2}}\left(g_{2} \tilde{\mathbf{x}}_{i, j}-g_{i, j} \tilde{\mathbf{x}}_{, 2}\right) \cdot \mathbf{N}
$$

Proof. The first coordinate of (3.19) is given by

$$
g_{i, j}=\Gamma_{i j}^{1} g_{1}+\Gamma_{i j}^{2} g_{2}
$$

Under assumption $g_{1} \neq 0$ we have

$$
\Gamma_{i j}^{1}=\frac{g_{i, j}-\Gamma_{i j}^{2} g_{2}}{g_{1}}
$$

and therefore (3.19) turns to

$$
\mathbf{x}_{, i, j}=\frac{1}{g_{1}}\left(g_{i, j} \mathbf{x}_{, 1}+W \Gamma_{i j}^{2} \sigma\right)+L_{i j} \mathbf{N}
$$

From (3.23) it follows that $g_{1} \mathbf{x}_{, i, j}-g_{i, j} \mathbf{x}_{, 1}$ is an isotropic vector equal to $W \Gamma_{i j}^{2} \sigma+L_{i j} g_{1} \mathbf{N}$. The coefficients $L_{i j}$ are obtained by scalar multiplication by $\mathbf{N}$.

In particular, for the parametrization (3.2) we have

$$
L_{11}=\epsilon \frac{1}{W}\left(0,0, z_{, x, x}\right) \cdot\left(0, z_{, y}, 1\right)=-\epsilon \frac{1}{W} z_{, x, x} \quad L_{12}=-\epsilon \frac{1}{W} z_{, x, y} \quad L_{22}=-\epsilon \frac{1}{W} z_{, y, y} .
$$

Functions $\Gamma_{i j}^{k}$ defined by (3.19) are called the Christoffel symbols of the second kind. Now we can prove the following proposition.

Proposition 3.4. Derivatives of the side tangential vector $\sigma$ and the normal vector $\mathbf{N}$ are given by

$$
\begin{aligned}
\sigma_{, i} & :=\frac{\partial}{\partial u_{i}} \sigma=\frac{1}{W}\left(g_{1} L_{i 2}-g_{2} L_{1 i}\right) \mathbf{N} . \\
\mathbf{N}_{, i} & =\frac{1}{W}\left(g_{1} L_{i 2}-g_{2} L_{1 i}\right) \sigma, \quad i=1,2 .
\end{aligned}
$$

Proof. Vectors $\sigma_{i}$ and $\mathbf{N}_{, i}$ are isotropic vectors and therefore can be expressed as linear combinations of $\sigma$ and $\mathbf{N}$. Since $\mathbf{N}^{2}:=\epsilon= \pm 1, \sigma^{2}=-\epsilon$ it follows $\mathbf{N} \cdot \mathbf{N}_{, i}=0, \sigma \cdot \sigma, i=0$. Also $\sigma_{, i} \cdot \mathbf{N}+\sigma \cdot \mathbf{N}_{, i}=0$. Having a pseudoscalar product in the isotropic plane, we conclude $\sigma_{, i}=f \mathbf{N}, \mathbf{N}_{, i}=f \sigma$, for a $C^{r}$-function $f, r \geq 1$.

Now, from the definition of $\sigma$ it follows that

$$
\sigma_{, i}=\left(\frac{g_{1}}{W}\right)_{, i} \mathbf{x}_{, 2}-\left(\frac{g_{2}}{W}\right)_{, i} \mathbf{x}_{, 1}+\frac{g_{1}}{W} \mathbf{x}_{, 2, i}-\frac{g_{2}}{W} \mathbf{x}_{, 1, i}
$$


By using (3.19) it is easy to show that the component by $\mathbf{N}$ of $\sigma_{, i}$ is equal to $(1 / W)\left(g_{1} L_{i 2}-\right.$ $\left.g_{2} L_{1 i}\right)$.

We will define the Gaussian curvature $K$ as the product of principal curvatures, the normal curvatures in the principal direction. The principal directions are tangent directions of a curve $c$ on a surface along which the normal field of the surface determines developable ruled surface, that is, $\operatorname{det}(\dot{c}, \mathbf{N}, \dot{N})=0$. This property characterizes principal directions in Euclidean space [16].

Proposition 3.5. The principal directions on a regular admissible surface $\Phi$ are given by

$$
g_{1} \mathrm{~d} u_{1}+g_{2} \mathrm{~d} u_{2}=0
$$

(the isotropic direction) and

$$
\left(g_{2} L_{11}-g_{1} L_{12}\right) \mathrm{d} u_{1}+\left(g_{2} L_{12}-g_{1} L_{22}\right) \mathrm{d} u_{2}=0
$$

Proof. Let $c(t)=(x(t), y(t), z(t))$ be a curve on a surface $\Phi$. Since $\mathbf{N}, \dot{\mathbf{N}}$ are isotropic vectors, $\operatorname{det}(\dot{c}, \mathbf{N}, \dot{\mathbf{N}})=0$ if and only if $\dot{x}=x_{, 1} \mathrm{~d} u_{1}+x_{, 2} \mathrm{~d} u_{2}=0$ which gives (3.27) or $\operatorname{det}(\tilde{\mathbf{N}}, \dot{\widetilde{\mathbf{N}}})=0$ which by using Proposition 3.4 gives (3.28).

The principal curvature is given in the next proposition.

Proposition 3.6. The principal curvature $k_{1}$ for the direction (3.27) is given by

$$
k_{1}=-\epsilon \frac{L_{11} g_{2}^{2}-2 L_{12} g_{1} g_{2}+L_{22} g_{1}^{2}}{W^{2}}
$$

and the principal curvature $k_{2}$ for the direction (3.28) by

$$
k_{2}=\frac{L_{11} L_{22}-L_{12}^{2}}{L_{11} g_{2}^{2}-2 L_{12} g_{1} g_{2}+L_{22} g_{1}^{2}} .
$$

Proof. Principal curvatures are calculated from $k=I I / I$, where $I I=L_{11} \mathrm{~d} u_{1}^{2}+2 L_{12} \mathrm{~d} u_{1} \mathrm{~d} u_{2}+$ $L_{22} \mathrm{~d} u_{2}^{2}$ is the second and $I=\mathrm{d} s^{2}$ the first fundamental form. Therefore, for the direction (3.27) we have

$$
\begin{aligned}
k_{1} & =\frac{L_{11} \mathrm{~d} u_{1}^{2}+2 L_{12} \mathrm{~d} u_{1} \mathrm{~d} u_{2}+L_{22} \mathrm{~d} u_{2}^{2}}{h_{11} \mathrm{~d} u_{1}^{2}+2 h_{12} \mathrm{~d} u_{1} \mathrm{~d} u_{2}+h_{22} \mathrm{~d} u_{2}^{2}} \\
& =\frac{L_{11} g_{2}^{2}-2 L_{12} g_{1} g_{2}+L_{22} g_{1}^{2}}{h_{11} g_{2}^{2}-2 h_{12} g_{1} g_{2}+h_{22} g_{1}^{2}}=-\epsilon \frac{L_{11} g_{2}^{2}-2 L_{12} g_{1} g_{2}+L_{22} g_{1}^{2}}{W^{2}}
\end{aligned}
$$


and for the direction (3.28)

$$
\begin{aligned}
k_{2} & =\frac{L_{11} \mathrm{~d} u_{1}^{2}+2 L_{12} \mathrm{~d} u_{1} \mathrm{~d} u_{2}+L_{22} \mathrm{~d} u_{2}^{2}}{\left(g_{1} \mathrm{~d} u_{1}+g_{2} \mathrm{~d} u_{2}\right)^{2}} \\
& =\frac{L_{11} L_{22}-L_{12}^{2}}{L_{11} g_{2}^{2}-2 L_{12} g_{1} g_{2}+L_{22} g_{1}^{2}} .
\end{aligned}
$$

The Gaussian curvature of a regular admissible surface is defined by

$$
K=k_{1} k_{2}=-\epsilon \frac{L_{11} L_{22}-L_{12}^{2}}{W^{2}}
$$

or for (3.2)

$$
K=-\epsilon \frac{z_{, x, x} z_{, y, y}-z_{, x, y}^{2}}{W^{4}}
$$

The mean curvature of a surface is defined by

$$
H=-\frac{\epsilon}{2 W^{2}}\left(g_{2}^{2} L_{11}-2 g_{1} g_{2} L_{12}+g_{1}^{2} L_{22}\right)=\frac{k_{1}}{2},
$$

and for (3.2) it turns to

$$
H=\frac{\epsilon}{2 W^{3}} z, y, y
$$

Such definition of $H$ is motivated by Proposition 3.7

The third fundamental form III is introduced in the analogous way as in Euclidean space. Since $\mathbf{N}$ is a unit isotropic field along $\Phi$, the end points of its associated position vectors lie on a hyperbolic unit sphere. More precisely, if $\mathbf{N}$ is a timelike (spacelike) field, that is, if a surface is spacelike (timelike), then the end points of associated position vectors of $\mathbf{N}$ lie on a unit spacelike sphere $z^{2}-y^{2}=1$ (unit timelike sphere $y^{2}-z^{2}=1$ ), see Figure 1 . The obtained mapping is called the Gauss map (the spherical map); the set of all end points of $\mathbf{N}$ is called the spherical image of a surface. The third fundamental form is the first fundamental form of the spherical image. Therefore it is defined by

$$
\begin{aligned}
I I I & =\mathrm{d} \mathbf{N} \cdot \mathrm{d} \mathbf{N}=\left(\mathbf{N}_{, 1} \mathrm{~d} u_{1}+\mathbf{N}_{, 2} \mathrm{~d} u_{2}\right)^{2} \\
& =\mathbf{N}_{, 1}^{2} \mathrm{~d} u_{1}^{2}+2 \mathbf{N}_{, 1} \cdot \mathbf{N}_{, 2} \mathrm{~d} u_{1} \mathrm{~d} u_{2}+\mathbf{N}_{, 2}^{2} \mathrm{~d} u_{2}^{2},
\end{aligned}
$$


where

$$
\begin{aligned}
\mathbf{N}_{, 1}^{2} & =-\frac{\epsilon}{W^{2}}\left(g_{2}^{2} L_{11}^{2}-2 g_{1} g_{2} L_{11} L_{12}+g_{1}^{2} L_{12}^{2}\right), \\
\mathbf{N}_{, 1} \cdot \mathbf{N}_{, 2} & =-\frac{\epsilon}{W^{2}}\left(g_{2}^{2} L_{11} L_{12}-g_{1} g_{2}\left(L_{11} L_{22}+L_{12}^{2}\right)+g_{1}^{2} L_{12} L_{22}\right), \\
\mathbf{N}_{, 2}^{2} & =-\frac{\epsilon}{W^{2}}\left(g_{2}^{2} L_{12}^{2}-2 g_{1} g_{2} L_{12} L_{22}+g_{1}^{2} L_{22}^{2}\right) .
\end{aligned}
$$

Particularly, for (3.2) we have $N=(1 / W)(0, z, y, 1)$, and

$$
I I I=-\epsilon\left(\mathrm{d}\left(\frac{z, y}{W}\right)^{2}-\mathrm{d}\left(\frac{1}{W}\right)^{2}\right)=-\epsilon\left(\frac{1}{W^{4}}\left(z_{, x, y} \mathrm{~d} x+z_{, y, y} \mathrm{~d} y\right)^{2}\right)
$$

By a simple computation we can notice that the following relation holds.

Proposition 3.7. One has $K I-2 H I I+I I I=0$.

Theorem 3.8. Minimal surfaces in a pseudo-Galielan space $G_{3}^{1}$ are ruled conoidal surfaces, that is, they are cones with vertices on the absolute line or ruled surfaces with the absolute line as a director curve in infinity.

Proof. We define a normal section in a point of a surface as a plane curve obtained as a section of the surface by a pseudo-Euclidean plane. It can be shown that the curvature of a normal section (parametrized by the arc-length) as a curve in a pseudo-Euclidean plane is $\kappa^{*}=k_{1}$. This is obtained from the fact that, for a curve $c(t)=\mathbf{x}(u(t), v(t))$ parametrized by the arc length, tangent vector field $c^{\prime}(t)$ is equal to the side tangential field $\sigma$, and therefore $c^{\prime \prime}(t)=$ $k_{1} \mathbf{N}$.

Furthermore, $\kappa^{*}=0$ if and only if a curve is a line in the pseudo-Euclidean plane, and therefore $c(t)$ is an isotropic line in $G_{3}^{1}$. Since $H=k_{1} / 2$, the assertion follows.

\section{Surfaces of Revolution}

In the pseudo-Galilean space $G_{3}^{1}$ there are two types of rotations: pseudo-Euclidean rotations given by the normal form

$$
\begin{aligned}
& \bar{x}=x, \\
& \bar{y}=y \cosh t+z \sinh t \\
& \bar{z}=y \sinh t+z \cosh t
\end{aligned}
$$


and isotropic rotations with the normal form

$$
\begin{aligned}
& \bar{x}(t)=x+b t \\
& \bar{y}(t)=y+x t+b \frac{t^{2}}{2} \\
& \bar{z}(t)=z,
\end{aligned}
$$

where $t \in \mathbf{R}$ and $b=$ const $>0$.

The trajectory of a single point under a pseudo-Euclidean rotation is a pseudoEuclidean circle (i.e., a rectangular hyperbola)

$$
x=\text { const., } \quad y^{2}-z^{2}=R^{2}, \quad R \in \mathbf{R} .
$$

The invariant $R$ is the radius of the circle. Pseudo-Euclidean circles intersect the absolute line $f$ in the fixed points of the hyperbolic involution $\left(F_{1}, F_{2}\right)$. There are three kinds of pseudoEuclidean circles: circles of real radius, of imaginary radius, and of radius zero. Circles of real radius are timelike curves (having timelike tangent vectors) and of imaginary radius spacelike curves (having spacelike tangent vectors).

The trajectory of a point under the isotropic rotation is an isotropic circle whose normal form is

$$
z=\text { const., } \quad y=\frac{x^{2}}{2 b}
$$

The invariant $b$ is the radius of the circle. The fixed line of the isotropic rotations (4.2) is the absolute line $f$.

By rotating a nonisotropic curve $u \mapsto(f(u), g(u), 0), g>0$, around the $x$-axis by pseudo-Euclidean rotations, we obtain a timelike surface

$$
\mathbf{x}(u, v)=(f(u), g(u) \cosh v, g(u) \sinh v),
$$

and by rotating a curve $u \mapsto(f(u), 0, g(u)), g>0$, around the $x$-axis, we obtain a spacelike surface

$$
\mathbf{x}(u, v)=(f(u), g(u) \sinh v, g(u) \cosh v) .
$$

The Gaussian curvature of these surfaces is given by $K=-\epsilon\left(g^{\prime} / f^{\prime}\right)^{\prime}\left(1 / f^{\prime} g\right)$ or if we assume that the rotated curve is parametrized by the arc length $u, u \mapsto(u, g(u), 0)(u \mapsto$ $(u, 0, g(u)))$ by

$$
K=-\epsilon \frac{g^{\prime \prime}}{g} .
$$


Therefore, surfaces with constant curvature $K_{0}$ are described by the ordinary differential equation

$$
g^{\prime \prime}+\epsilon K_{0} g=0, \quad K_{0}=\text { const. }
$$

Their implicit equation is

$$
y^{2}-z^{2}=\epsilon g^{2}(x)
$$

and the first fundamental form

$$
\mathrm{d} s^{2}=\mathrm{d} u^{2}-\epsilon \delta g^{2}(u) \mathrm{d} v^{2} .
$$

The following theorem holds.

Theorem 4.1. The profile curve of surfaces of revolution of constant Gaussian curvature in pseudoGalilean space is as follows.

(1) If $K=-\epsilon 1 / a^{2}$ (i.e., $K=1 / a^{2}$ for spacelike surfaces and $K=-1 / a^{2}$ for timelike surfaces), then the general solution of the differential equation (4.8) is

$$
g(u)=A \sinh \left(\frac{u}{a}\right)+B \cosh \left(\frac{u}{a}\right), \quad A, B, a \in \mathbf{R}
$$

(2) If $K=0$, then the general solution of the differential equation (4.8) is

$$
g(u)=A u+B, \quad A, B \in \mathbf{R} .
$$

(3) If $K=\epsilon 1 / a^{2}$, then the general solution of the differential equation (4.8) is

$$
g(u)=A \sin \left(\frac{u}{a}\right)+B \cos \left(\frac{u}{a}\right), \quad A, B, a \in \mathbf{R} .
$$

Examples of these surfaces are given in Figures 2 and 3. Notice that if $K=0$, then the profile curve is a line (4.12). Among these surfaces there are also hyperbolic spheres $(A=0$, $\left.y^{2}-z^{2}= \pm B^{2}\right)$, see Figures 1 and 4 . Cones $\left(A \neq 0, y^{2}-z^{2}= \pm(A x+B)^{2}\right)$ are also surfaces of revolution with vanishing curvature.

Next we consider the isotropic rotations. By rotating an isotropic curve $u \mapsto$ $(0, f(u), g(u))$ about the $z$-axis by isotropic rotation, we obtain a surface

$$
\mathbf{x}(u, v)=\left(v, f(u)+\frac{v^{2}}{2 b^{\prime}}, g(u)\right), \quad b \neq 0
$$

Let us assume that the rotated curve is parametrized by the arc length

$$
f^{\prime 2}-g^{\prime 2}=-\epsilon
$$




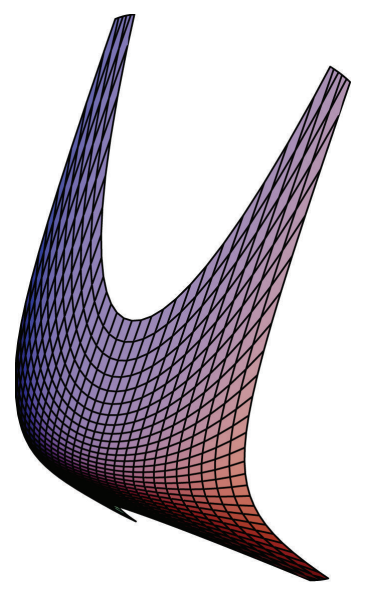

Figure 2: A spacelike (timelike) surface of revolution with positive (negative) curvature.

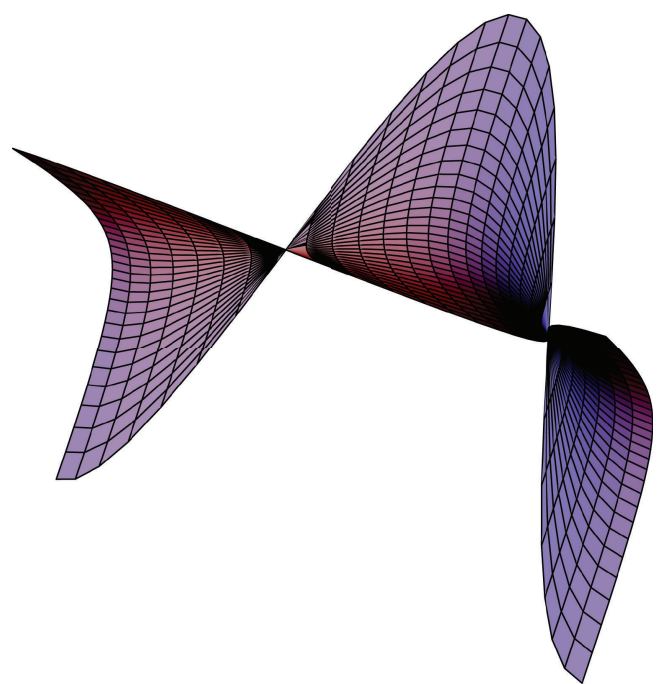

Figure 3: A spacelike (timelike) surface of revolution with negative (positive) curvature.

that is, the curve is spacelike (its tangent vectors are spacelike, $\epsilon=-1$ ) or timelike (its tangent vectors are timelike, $\epsilon=1$ ). By a simple calculation it can be shown that by revolving a spacelike (timelike) curve a spacelike (timelike) surface is obtained. From (4.15) it follows $g^{\prime \prime}=\left(f^{\prime} / g^{\prime}\right) f^{\prime \prime}$, and therefore, the following expression for $K$ is obtained regardless of the type of the surface:

$$
K=-\frac{f^{\prime \prime}}{b}=K_{0}=\text { const } .
$$



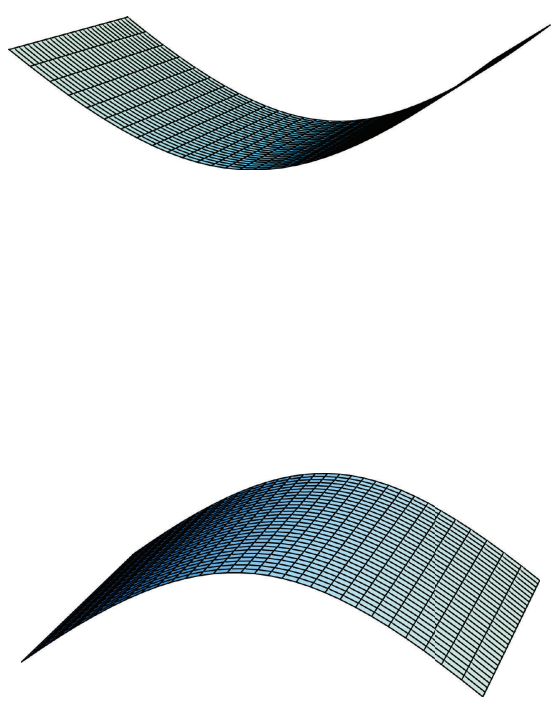

Figure 4: A spacelike hyperbolic sphere $(K=0)$.

Therefore, the profile curve of a surface with constant curvature is described by the ordinary differential equation

$$
f^{\prime \prime}=-b K_{0}
$$

Theorem 4.2. The profile curve $u \mapsto(0, f(u), g(u))$ of a surface with constant curvature obtained by isotropic rotations is given by

$$
\begin{gathered}
f(u)=\frac{-b K_{0} u^{2}}{2}+A u+B, \quad A, B \in \mathbf{R}, \\
g(u)=-\frac{1}{2 b K_{0}}\left(\left(A-b K_{0} u\right) \sqrt{\left(A-b K_{0} u\right)^{2}-1}-\operatorname{Arccosh}\left(A-b K_{0} u\right)+C\right),
\end{gathered}
$$

for a spacelike surface and

$$
g(u)=-\frac{1}{2 b K_{0}}\left(\left(A-b K_{0} u\right) \sqrt{\left(A-b K_{0} u\right)^{2}+1}+\operatorname{Arcsinh}\left(A-b K_{0} u\right)+C\right)
$$

and for a timelike surface, where $C \in \mathbf{R}$ is a constant.

If $K_{0}=0$, then

$$
f(u)=A u+B, \quad g(u)=C u+D, \quad A, B, C, D \in \mathbf{R}, \quad A^{2}-C^{2}=-\epsilon
$$

which implies that the profile curve is a line and obtained surface a parabolic sphere $\mathbf{x}(u, v)=$ $\left(b v,(A u+B)+b v^{2} / 2, C u+D\right)($ Figure 5). 


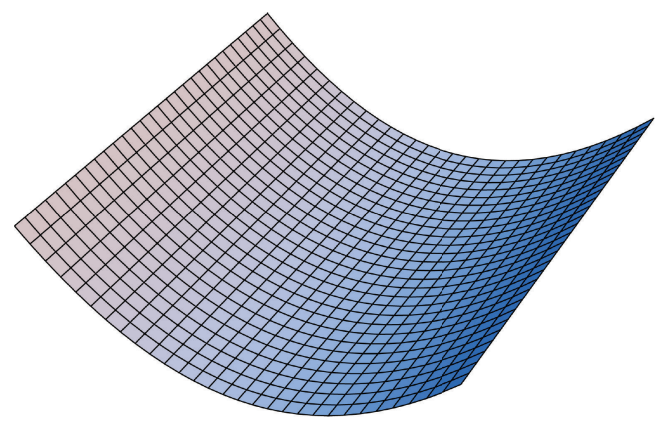

Figure 5: A spacelike parabolic sphere $(K=0)$.

We can notice that this situation appears much more simpler than the same situation in the Euclidean space, where the expressions of the profile curves involve elliptic integrals.

Now we treat surfaces of constant mean curvature. The mean curvature $H$ of the surfaces $(4.5),(4.6)$ is given by

$$
H=-\frac{\epsilon}{2 W^{2}}=\frac{1}{2 g}
$$

Therefore the following theorem holds.

Theorem 4.3. There are no minimal surfaces of revolution (4.5), (4.6). Surfaces with constant mean curvature $H \neq 0$ are hyperbolic timelike, respectively, spacelike spheres obtained by rotating a line $(u,-1 / 2 H, 0)$, resp., $(u, 0,-(1 / 2 H))$.

The mean curvature $H$ of a surface (4.14) is given by

$$
H=-\frac{\epsilon}{2} \frac{f^{\prime \prime}}{g^{\prime}}=-\frac{\epsilon}{2} \frac{f^{\prime \prime}}{\sqrt{f^{\prime 2}+\epsilon}} .
$$

Theorem 4.4. The profile curve of a surface of revolution of constant mean curvature obtained by isotropic rotations (Figure 6) in pseudo-Galilean space is as follows.

(1) If $H=0$, then $f(u)=A u+B, g(u)=A_{1} u+B_{1}, A, B, A_{1}, B_{1}, A^{2}-A_{1}^{2}=-\epsilon$, that is, the surface is generated by an isotropic rotation of an isotropic line (a parabolic sphere).

(2) If $H \neq 0$, then for a spacelike surface $(\epsilon=-1)$

$$
f(u)=\frac{1}{h_{0}} \sinh \left(h_{0} u+c\right)+c_{1}, \quad g(u)=\frac{1}{h_{0}} \cosh \left(h_{0} u+c\right)+c_{2}
$$

and for a timelike surface $(\epsilon=1)$

$$
f(u)=\frac{1}{h_{0}} \cosh \left(h_{0} u+c\right)+c_{1}, \quad g(u)=\frac{1}{h_{0}} \sinh \left(h_{0} u+c\right)+-2,
$$




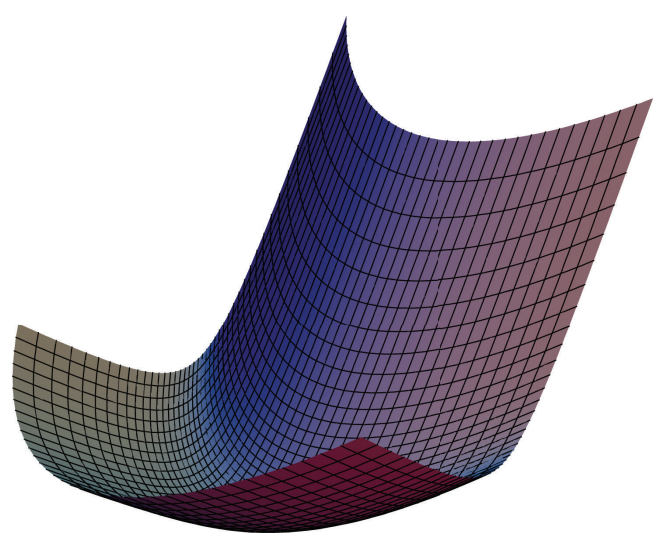

Figure 6: A surface with constant mean curvature obtained by an isotropic rotation.

where $h_{0}= \pm 2 H \epsilon=$ const, $c, c_{1}, c_{2}=$ const. A surface is obtained by an isotropic rotation of a pseudo-Euclidean circle.

\section{Klein-Gordon Equation and the Tchebyshev Coordinates in $G_{3}^{1}$}

In the context of classical surface theory in the three-dimensional Euclidean space, the sine-Gordon equation has the geometrical interpretation in terms of surfaces with negative constant Gaussian curvature. This is shown by parametrizing a surface by coordinates that satisfy

$$
E=G=1, \quad F=\cos \varphi,
$$

where $E, F$, and $G$ are coefficients of the first fundamental form (i.e., by Tchebyshev coordinates). Then Theorema Egregium implies

$$
\varphi_{, u, v}=-K \sin \varphi \text {. }
$$

In particular, for a surface with constant negative curvature $K=-1 / m^{2}$, the previous equation turns to the sine-Gordon equation for the angle $\varphi$ between parametric curves

$$
\varphi, u, v=\frac{1}{m^{2}} \sin \varphi
$$

Similar results hold in a three-dimensional pseudo-Riemannian manifold of constant curvature (e.g., Minkowski space $\mathbf{R}_{3}^{1}$ ). The angle between curves of the Tchebyshev net on a spacelike (timelike) surface of constant negative (positive) curvature satisfies the sineGordon equation or its hyperbolic analogue sinh-Gordon equation [17].

Our aim is to introduce the analogue of the Tchebyshev coordinates on a surface in the pseudo-Galilean space and to establish a partial differential equation satisfied by the angle between the parametric curves. In order to be able to consider the angle between parametric 
curves of a surface, it is assumed that parametric curves are non-isotropic curves, that is, $g_{1} \neq 0, g_{2} \neq 0$.

We proceed with the following definition.

Definition 5.1. Tchebyshev net on a surface is the net of parametric curves for which the first fundamental coefficients satisfy $g_{1}=g_{2}=1, h_{11,2}=h_{22,1}$.

Notice that according to our notation we have $h_{i i, j}=\partial h_{i i} / \partial u_{j}, i, j=1,2$.

The first condition from the definition implies that the parametric curves of this net are parametrized by the arc length. The side tangential vector in these coordinates is given by $\sigma=(1 / W)\left(\mathbf{x}_{, 2}-\mathbf{x}_{, 1}\right)$, where $W^{2}=-\epsilon\left(\mathbf{x}_{, 2}-\mathbf{x}_{, 1}\right)^{2}$. Furthermore, since

$$
h_{11,2}=\frac{\partial \widetilde{\mathbf{x}}_{, 1}^{2}}{\partial v}=2 \mathbf{x}_{, 1} \cdot \mathbf{x}_{, 1,2,} \quad h_{22,1}=\frac{\partial \widetilde{\mathbf{x}}_{, 2}^{2}}{\partial u}=2 \mathbf{x}_{, 2} \cdot \mathbf{x}_{, 1,2}
$$

it follows that

$$
\sigma \cdot \mathbf{x}_{, 1,2}=\frac{1}{W}\left(\mathbf{x}_{, 2}-\mathbf{x}_{, 1}\right) \cdot \mathbf{x}_{, 1,2}=\frac{1}{2 W}\left(h_{22,1}-h_{11,2}\right)=0 .
$$

Such definition of the Tchebyshev coordinates is motivated by the following theorem whose counterpart holds in Euclidean space. For the analogous result in simply isotropic space $I_{3}^{1}$ see [18].

Theorem 5.2. Asymptotic curves on a $C^{r}$-surface $\Phi$ in $G_{3}^{1}, r \geq 3$, form the Tchebychev net if and only if $\Phi$ is a spacelike surface with constant negative curvature or timelike surface with constant positive curvature.

Proof. First notice that on spacelike surfaces with negative curvature and timelike surface with positive curvature there are two families of real asymptotic curves, due to the fact that the equation for the asymptotic curves $I I=0$ has two real solutions.

Proof follows from the analogues of the Gauss and Codazzi-Mainardi equations for surfaces in the pseudo-Galilean space $G_{3}^{1}$. They are obtained in the following way.

Let $\mathbf{x}=\mathbf{x}\left(x\left(u_{1}, u_{2}\right), y\left(u_{1}, u_{2}\right), z\left(u_{1}, u_{2}\right)\right)$ be a parametrization of $\Phi$, and let $\mathbf{N}$ denote its unit normal field. Multiplying (3.23) by $\sigma$ (and analogous expression obtained when assuming $\left.g_{2} \neq 0\right)$, it can be shown that the Christoffel symbols of the second kind defined by (3.19) are given by

$$
\begin{aligned}
\Gamma_{i j}^{1} & =\frac{\epsilon}{W}\left(g_{2} \tilde{\mathbf{x}}_{i, j}-g_{i, j} \tilde{\mathbf{x}}_{, 2}\right) \cdot \sigma, \\
\Gamma_{i j}^{2} & =-\frac{\epsilon}{W}\left(g_{1} \tilde{\mathbf{x}}_{, i, j}-g_{i, j} \tilde{\mathbf{x}}_{, 1}\right) \cdot \sigma .
\end{aligned}
$$


Under assumption $\mathbf{x}_{, i, j, k}=\mathbf{x}_{, i, k, j}$ the following is obtained:

$$
\begin{aligned}
\left(L_{i j, k}\right. & \left.-L_{i k, j}+\sum_{n}\left(\Gamma_{i j}^{n} L_{n k}-\Gamma_{i k}^{n} L_{n j}\right)\right) \mathbf{N} \\
& +\sum_{m}\left(\Gamma_{i j, k}^{m}-\Gamma_{i k, j}^{m}+\sum_{n}\left(\Gamma_{i j}^{n} \Gamma_{n k}^{m}-\Gamma_{i k}^{n} \Gamma_{n j}^{m}+g^{m} g^{n}\left(L_{i j} L_{n k}-L_{i k} L_{j n}\right)\right)\right) \mathbf{x}_{, m}=\mathbf{0}
\end{aligned}
$$

where $g^{1}=g_{2} / W, g^{2}=-g_{1} / W$. We can notice that the previous formula differs from its analogue in the Galilean space [10] in the sign of the term $g^{m} g^{n}\left(L_{i j} L_{n k}-L_{i k} L_{j n}\right)$. This is a consequence of the formulas for $\mathbf{N}_{i}$ in Proposition 3.4 (with the opposite sign than in the Galilean space).

The component by $\mathbf{x}_{, m}$ gives the Gauss (integrability) equation

$$
\begin{aligned}
\Gamma_{i j, k}^{m} & -\Gamma_{i k, j}^{m}+\sum_{n}\left(\Gamma_{i j}^{n} \Gamma_{n k}^{m}-\Gamma_{i k}^{n} \Gamma_{n j}^{m}\right) \\
& =-\sum_{n} g^{m} g^{n}\left(L_{i j} L_{n k}-L_{i k} L_{j n}\right), \quad m=1,2,
\end{aligned}
$$

and the component by $\mathbf{N}$ the Codazzi-Mainardi equation

$$
L_{i j, k}-L_{i k, j}=-\sum_{n}\left(\Gamma_{i j}^{n} L_{n k}-\Gamma_{i k}^{n} L_{n j}\right)
$$

Now, let us assume that the Gaussian curvature $K$ of a surface is constant and that the parametric curves asymptotic. For parametrization with asymptotic lines we have $L_{11}=$ $L_{22}=0$. We consider spacelike surfaces with negative curvature and timelike surfaces with positive curvature, that is, surfaces that have two families of asymptotic lines. In asymptotic coordinates equation (5.9) for $i=j=1, k=2$, and $i=k=2, j=1$ reduces to

$$
\begin{aligned}
& L_{12,1}=L_{12}\left(\Gamma_{11}^{1}-\Gamma_{12}^{2}\right), \\
& L_{12,2}=L_{12}\left(\Gamma_{22}^{2}-\Gamma_{12}^{1}\right) .
\end{aligned}
$$

Furthermore, the assumption $K=$ const. $=\epsilon\left(1 / m^{2}\right)$ implies $L_{12}^{2}=W^{2}\left(1 / m^{2}\right)$. By derivating this equation with using (5.10) we get

$$
\begin{aligned}
& W_{, 1}=W\left(\Gamma_{11}^{1}-\Gamma_{12}^{2}\right), \\
& W_{, 2}=W\left(\Gamma_{22}^{2}-\Gamma_{12}^{1}\right) .
\end{aligned}
$$


On the other hand, partial derivatives of $W, W^{2}=-\epsilon\left(g_{1} \mathbf{x}_{, 2}-g_{2} \mathbf{x}_{1,1}\right)^{2}$ for an arbitrary parametrization of a regular admissible surface are equal to

$$
\begin{aligned}
& W_{, 1}=W\left(\Gamma_{11}^{1}+\Gamma_{12}^{2}\right), \\
& W_{, 2}=W\left(\Gamma_{22}^{2}+\Gamma_{12}^{1}\right) .
\end{aligned}
$$

They are obtained by derivating the expression $W^{2}=-\epsilon\left(g_{1} \mathbf{x}_{, 2}-g_{2} \mathbf{x}_{, 1}\right)^{2}$ and using

$$
\begin{aligned}
& g_{2} \mathbf{x}_{, 1,1} \cdot \sigma=\frac{W}{\epsilon} \Gamma_{11}^{1}+g_{1,1} \mathbf{x}_{, 2} \cdot \sigma \\
& g_{1} \mathbf{x}_{, 1,2} \cdot \sigma=-\frac{W}{\epsilon} \Gamma_{12}^{2}+g_{1,2} \mathbf{x}_{, 1} \cdot \sigma
\end{aligned}
$$

obtained from (5.6) for $i=j=1$ and $i=1, j=2$.

Now from (5.11) and (5.12) it follows that

$$
\Gamma_{12}^{1}=\Gamma_{12}^{2}=0
$$

By using expressions in (5.6) we can write this system as

$$
\begin{aligned}
& \left(g_{1,2} \mathbf{x}_{, 1}-g_{1} \mathbf{x}_{, 1,2}\right) \cdot \sigma=0, \\
& \left(g_{2} \mathbf{x}_{1,1,2}-g_{1,2} \mathbf{x}_{, 2}\right) \cdot \sigma=0 .
\end{aligned}
$$

It follows that

$$
g_{1,2}\left(g_{2} \mathbf{x}_{, 1}-g_{1} \mathbf{x}_{, 2}\right) \cdot \sigma=0 \text {. }
$$

Furthermore $\left(g_{2} \mathbf{x}_{11}-g_{1} \mathbf{x}_{2,2}\right) \cdot \sigma=-W \sigma^{2} \neq 0$, and therefore the previous equation implies

$$
g_{1,2}=0 .
$$

Substituting (5.17) into the system (5.15) we obtain (since $g_{i} \neq 0, i=1,2$ )

$$
\mathbf{x}_{, 1,2} \cdot \sigma=0
$$

Let us analyze conditions (5.17), (5.18). Condition (5.17) implies $g_{1}=g_{1}(u), g_{2}=g_{2}(v)$, that is, functions $g_{i}$ are functions of one parameter only.

Condition (5.18) implies

$$
\mathbf{x}_{, 1,2} \cdot \sigma=\frac{1}{W} \mathbf{x}_{1,2} \cdot\left(g_{1} \mathbf{x}_{, 2}-g_{2} \mathbf{x}_{, 1}\right)=\frac{1}{2 W}\left(g_{1} h_{22,1}-g_{2} h_{11,2}\right),
$$


and therefore

$$
g_{1} h_{22,1}-g_{2} h_{11,2}=0
$$

Condition (5.17) enables us to introduce new coordinates

$$
\bar{u}=\int g_{1} \mathrm{~d} u, \quad \bar{v}=\int g_{2} \mathrm{~d} v
$$

to obtain $\bar{g}_{1}=d x / d \bar{u}=(d x / d u)(d u / d \bar{u})=g_{1}\left(1 / g_{1}\right)=1$. Analogously we can obtain $\bar{g}_{2}=1$. For the coefficients $\bar{h}_{i j}$ of the new parametrization we have

$$
\begin{aligned}
& \bar{h}_{11}=\left(\frac{\partial \tilde{\mathbf{x}}}{\partial \bar{u}}\right)^{2}=\frac{1}{g_{1}^{2}} h_{11} \\
& \bar{h}_{22}=\left(\frac{\partial \tilde{\mathbf{x}}}{\partial \bar{v}}\right)^{2}=\frac{1}{g_{2}^{2}} h_{22} .
\end{aligned}
$$

Therefore, since $g_{1,2}=0$,

$$
h_{11,2}=g_{2} g_{1}^{2} \bar{h}_{11,2}, \quad h_{22,1}=g_{1} g_{2}^{2} \bar{h}_{22,1} \text {. }
$$

Condition (5.20) now implies $\left(\partial \bar{h}_{11} / \partial \bar{v}\right)-\left(\partial \bar{h}_{22} / \partial \bar{u}\right)=\bar{h}_{11,2}-\bar{h}_{22,1}=\left(1 / g_{2} g_{1}^{2}\right) h_{11,2}-$ $\left(1 / g_{1} g_{2}^{2}\right) h_{22,1}=\left(1 / g_{1}^{2} g_{2}^{2}\right)\left(g_{2} h_{11,2}-g_{1} h_{22,1}\right)=0$, which means that the considered coordinates are Tchebychev.

Conversely, if the asymptotic curves form the Tchebychev net, let us prove that $\Phi$ has constant curvature. The assumptions imply $L_{11}=L_{22}=0$ and

$$
\mathbf{x}_{, 1,2} \cdot \sigma=0
$$

where $\sigma=(1 / w)\left(\mathbf{x}_{, 2}-\mathbf{x}_{, 1}\right)$. Expressions in (5.6) imply that in Tchebyshev coordinates Christoffel symbols $\Gamma_{12}^{1}, \Gamma_{12}^{2}$, are given by

$$
\begin{gathered}
\Gamma_{12}^{1}=\frac{\epsilon}{W} \mathbf{x}_{, 1,2} \cdot \sigma=0, \\
\Gamma_{12}^{2}=-\frac{\epsilon}{W} \mathbf{x}_{, 1,2} \cdot \sigma=0 .
\end{gathered}
$$

Therefore Codazzi-Mainardi equations (5.10) are equal to

$$
L_{12,1}=L_{12} \Gamma_{11}^{1}, \quad L_{12,2}=L_{12} \Gamma_{22}^{2} .
$$


Now by differentiating

$$
K=\epsilon \frac{L_{12}^{2}}{W^{2}}
$$

partially in the first variable, we obtain

$$
K_{, 1} W^{3}=2 \epsilon L_{12}\left(L_{12,1} W-L_{12} W_{, 1}\right)
$$

and (5.26) implies

$$
K_{, 1} W^{3}=2 \epsilon L_{12}^{2}\left(\Gamma_{11}^{1} W-W, 1\right) .
$$

Since from (5.12) we have

$$
W_{, 1}=\Gamma_{11}^{1} W,
$$

it follows that

$$
K_{, 1}=0 .
$$

In the same way we can conclude

$$
K_{, 2}=0 \text {, }
$$

and therefore $K=$ const, what was claimed.

Let us now determine the angle between curves of the Tchebyshev net on a regular admissible surface. The angle between nonisotropic unit vectors

$$
\mathbf{a}=\left(1, a_{2}, a_{3}\right), \quad \mathbf{b}=\left(1, b_{2}, b_{3}\right)
$$

in the pseudo-Galilean space $G_{3}^{1}$ is determined by using the following expression:

$$
\varphi^{2}=\angle(\mathbf{a}, \mathbf{b})^{2}=\left|\left(b_{2}-a_{2}\right)^{2}-\left(b_{3}-a_{3}\right)^{2}\right|
$$

The defined angle is invariant under the group of motions (2.2). By applying expression (5.34) to the tangent vectors of the Tchebyshev parametric curves, we obtain

$$
\varphi^{2}=\left|\left(y_{2}-y_{1}\right)^{2}-\left(z_{2}-z_{1}\right)^{2}\right|=W^{2},
$$


and therefore we can write

$$
\varphi^{2}=-\epsilon\left(\left(y_{2}-y_{1}\right)^{2}-\left(z_{2}-z_{1}\right)^{2}\right)
$$

The function $\varphi$ and the function $W$ as well are differentiable of class $C^{r}, r \geq 2$, if and only if a surface is not lightlike.

For the Tchebyshev coordinates we have

$$
g_{1}=g_{2}=1, \quad \Gamma_{12}^{1}=\Gamma_{12}^{2}=0,
$$

and the Gauss (integrability) equation (5.8) for $i=j=1, k=2, m=1$ turns to

$$
\Gamma_{11,2}^{1}+\Gamma_{11}^{2} \Gamma_{22}^{1}=-\frac{L_{12}^{2}}{W^{2}}=-\epsilon K
$$

Furthermore, from (5.12) we have

$$
\Gamma_{11}^{1}=\frac{W_{, 1}}{W}, \quad \Gamma_{22}^{2}=\frac{W_{, 2}}{W},
$$

which with (5.6) implies

$$
\Gamma_{11}^{2}=-\frac{W_{, 1}}{W}, \quad \Gamma_{22}^{1}=-\frac{W_{, 2}}{W} .
$$

Therefore

$$
-\epsilon K=\frac{W W_{, 1,2}-W_{, 1} W_{, 2}}{W^{2}}+\left(-\frac{W_{, 1}}{W}\right)\left(-\frac{W_{, 2}}{W}\right)=\frac{W_{, 1,2}}{W}
$$

that is, $W, 1,2=-\epsilon K W$. Since $W= \pm \varphi$, where $\varphi$ is the angle between Tchebyshev curves, then we have

$$
\varphi, 1,2=-\epsilon K \varphi
$$

If the Gaussian curvature of the surface is constant $K=\epsilon\left(1 / m^{2}\right), m \in \mathbf{R}$, the previous equation turns to the Klein-Gordon equation

$$
\varphi, 1,2=-\frac{1}{m^{2}} \varphi
$$

Therefore we have proved the following theorem:

Theorem 5.3. The angle between curves of the Tchebychev net and the function $W$ as well on a spacelike surface of constant negative curvature and timelike surface of constant positive curvature in $G_{3}^{1}$ satisfy the Klein-Gordon equation. 
Finally, the functions

$$
\kappa(u, v)=\sqrt{\left|\mathbf{x}_{, 1,1}(u, v) \cdot \mathbf{x}_{, 1,1}(u, v)\right|}, \quad \overline{\mathcal{\kappa}}(u, v)=\sqrt{\left|\mathbf{x}_{, 2,2}(u, v)\right| \cdot \mathbf{x}_{, 2,2}(u, v)}
$$

satisfy the Klein-Gordon equation as well. The function $\kappa\left(u, v_{0}\right)$ is the curvature of a parametric curve $v=v_{0}$ of the Tchebychev net and $\bar{\kappa}\left(u_{0}, v\right)$ a parametric curve $u=u_{0}$. Notice that these curves are parametrized by the arc-length, since $x_{1}=x_{2}=1$.

Theorem 5.4. The functions $\kappa, \bar{\kappa}$ on a spacelike surface of constant negative curvature and timelike surface of constant positive curvature in $G_{3}^{1}$ satisfy the Klein-Gordon equation.

Proof. We have

$$
\mathbf{x}_{1,1}=\Gamma_{11}^{1}\left(\mathbf{x}_{, 1}-\mathbf{x}_{2,2}\right)=\Gamma_{11}^{1}(-W \sigma)=-W_{, 1} \sigma
$$

and therefore $\mathbf{x}_{1,1}$ is a spacelike (timelike) vector for a spacelike (timelike) surface. Hence we can write

$$
\kappa^{2}(u, v)=-\epsilon \mathbf{x}_{, 1,1} \cdot \mathbf{x}_{, 1,1}=W_{, 1}^{2} .
$$

Now we have

$$
\kappa \kappa_{, 1}=W_{, 1} W_{, 1,1}, \quad \kappa \kappa_{, 2}=W_{, 1} W_{, 1,2}
$$

Therefore

$$
\begin{aligned}
\mathcal{\kappa} \mathcal{\kappa}_{, 1,2} & =\left(\kappa \kappa_{, 2}\right)_{, 1}-\kappa_{, 1} \kappa_{, 2} \\
& =\left(W_{, 1} W_{, 1,2}\right)_{, 1}-W_{, 1,1} W_{, 1,2}=W_{, 1} W_{, 1,2,1}=-\frac{1}{m^{2}} W_{, 1}^{2}=-\frac{1}{m^{2}} \kappa^{2} .
\end{aligned}
$$

Therefore we have $\kappa, 1,2=-\left(1 / m^{2}\right) \mathcal{\kappa}$. Analogously we prove for $\overline{\mathcal{\kappa}}$.

Theorem 5.5. The parametric net on a spacelike surface of revolution (4.6) obtained by pseudoEuclidean rotations forms the Tchebyshev net in the following parametrization of the surface ( $K=$ $\left.-1 / a^{2}\right)$ :

$$
\begin{aligned}
& x(u, v)=u+v \\
& y(u, v)=\left(A \sin \left(\frac{u+v}{a}\right)+B \cos \left(\frac{u+v}{a}\right)\right) \sinh \left(\frac{u-v}{a}\right), \\
& z(u, v)=\left(A \sin \left(\frac{u+v}{a}\right)+B \cos \left(\frac{u+v}{a}\right)\right) \cosh \left(\frac{u-v}{a}\right)
\end{aligned}
$$


and on a timelike surface of revolution $(4.5)\left(K=1 / a^{2}\right.$, see Figure 7$)$

$$
\begin{aligned}
& x(u, v)=u+v \\
& y(u, v)=\left(A \sin \left(\frac{u+v}{a}\right)+B \cos \left(\frac{u+v}{a}\right)\right) \cosh \left(\frac{u-v}{a}\right) \\
& z(u, v)=\left(A \sin \left(\frac{u+v}{a}\right)+B \cos \left(\frac{u+v}{a}\right)\right) \sinh \left(\frac{u-v}{a}\right) .
\end{aligned}
$$

The parametric net on a surface of revolution (4.14) obtained by isotropic rotations forms the Tchebyshev net in the following parametrization of a spacelike surface with $K=-2 a / b<0$, $a, b \in \mathbf{R} \backslash\{0\}$ (see Figure 8):

$$
\begin{aligned}
x(u, v)= & u+v \\
y(u, v)= & \frac{1}{4 a}\left(-A+\cosh \left(\sqrt{\frac{2 a}{b}}(u-v)\right)\right)^{2}+\frac{A}{2 a}\left(-A+\cosh \left(\sqrt{\frac{2 a}{b}}(u-v)\right)\right) \\
& +B+\frac{1}{2 b}(u+v)^{2}, \\
z(u, v)= & \frac{1}{4 a}\left(\frac{1}{2} \sinh \left(\sqrt{\frac{2 a}{b}}(2 u-2 v)\right)-\sqrt{\frac{2 a}{b}}(u-v)\right),
\end{aligned}
$$

and of a timelike surface with $K=(2 a / b)>0, a, b \in \mathbf{R} \backslash\{0\}$

$$
\begin{aligned}
x(u, v)= & u+v \\
y(u, v)= & -\frac{1}{4 a}\left(A-\sinh \left(\sqrt{\frac{2 a}{b}}(u-v)\right)\right)^{2}+\frac{A}{2 a}\left(A-\sinh \left(\sqrt{\frac{2 a}{b}}(u-v)\right)\right) \\
& +B+\frac{1}{2 b}(u+v)^{2} \\
z(u, v)= & \frac{1}{4 a}\left(\frac{1}{2} \sinh \left(\sqrt{\frac{2 a}{b}}(2 u-2 v)\right)+\sqrt{\frac{2 a}{b}}(u-v)\right) .
\end{aligned}
$$

Remark 5.6. Notice that for the given parametrizations of surfaces obtained by pseudoEuclidean rotations we have

$$
g_{1}=g_{2}=1, \quad h_{11}=h_{22}
$$

that is, Tchebyshev curves satisfy condition similar to that one in the Euclidean space ( $E=$ $G=1)$. Therefore, the parametric curves satisfy $h_{11,2}=h_{22,1}=h_{11,1}$ and $h_{22,1}=h_{11,2}=h_{22,2}$, 


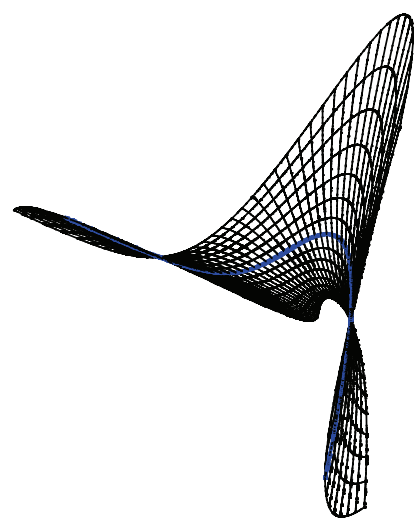

(a)

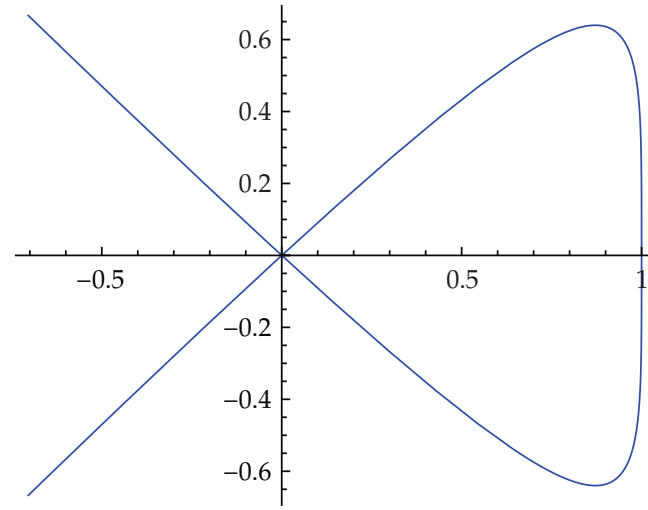

(b)

Figure 7: A timelike surface obtained by pseudo-Euclidean rotations parametrized by the Tchebychev net and the projection of a parametric curve onto $y z$-plane.

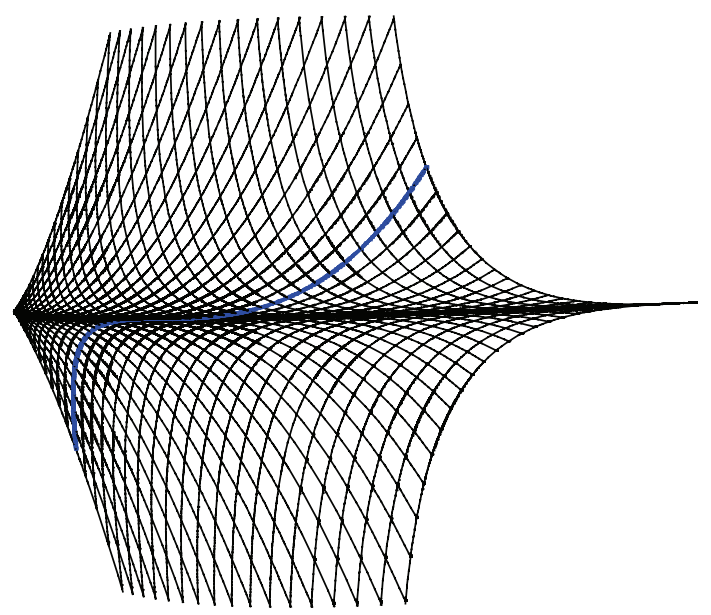

(a)

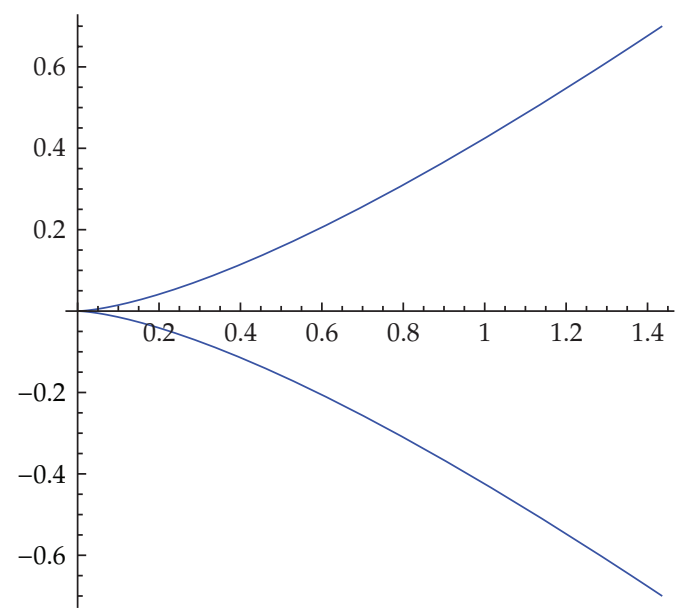

(b)

Figure 8: A spacelike surface obtained by isotropic rotations parametrized by the Tchebychev net and the projection of a parametric curve onto $y z$-plane.

which describes $h_{11}, h_{22}$ as the functions of form $\psi(u+v)$. The angle between curves of the Tchebychev net for spacelike and timelike surfaces obtained by pseudo-Euclidean rotations is equal to

$$
\varphi(u, v)=\frac{2}{a}\left(A \sin \left(\frac{u+v}{a}\right)+B \cos \left(\frac{u+v}{a}\right)\right)
$$

For spacelike surfaces obtained by isotropic rotations we have

$$
h_{11}-h_{22}=\frac{u+v}{2 a b^{2}},
$$


and the angle is equal to

$$
\varphi(u, v)=\sqrt{\frac{2}{a b}} \sinh \sqrt{\frac{2 a}{b}}(u-v)
$$

and for timelike surfaces obtained by isotropic rotations to

$$
\varphi(u, v)=\sqrt{\frac{2}{a b}} \cosh \sqrt{\frac{2 a}{b}}(u-v) .
$$

Furthermore, functions $\kappa(u, v), \bar{\kappa}(u, v)$ are given by

$$
\kappa(u, v)=\bar{\kappa}(u, v)=\frac{2}{a^{2}}\left(A \cos \left(\frac{u+v}{a}\right)-B \sin \left(\frac{u+v}{a}\right)\right)
$$

for spacelike and timelike surfaces obtained by pseudo-Euclidean rotations and by

$$
\kappa(u, v)=\bar{\kappa}(u, v)=\frac{2}{b} \cosh \sqrt{\frac{2 a}{b}}(u-v), \quad \kappa(u, v)=\bar{\kappa}(u, v)=\frac{2}{b} \sinh \sqrt{\frac{2 a}{b}}(u-v)
$$

for spacelike, respectively, timelike surfaces obtained by isotropic rotations.

Remark 5.7. Notice that the torsion of the parametric curves in the Tchebyshev parametrization is constant. This is the consequence of the general result which states that the torsion of asymptotic curves on a spacelike surface of negative curvature and timelike surface of positive curvature is equal to $\tau= \pm \sqrt{\epsilon K}$.

Remark 5.8. From a known solution of the Klein-Gordon equation, we can construct a surface. Let us consider a more general particular solution of the Klein-Gordon equation, that is, the solution

$$
\varphi(u, v)=e^{\lambda(u-v) / a}\left(A \cos \left(\frac{\mu(u+v)}{a}\right)+B \sin \left(\frac{\mu(u+v)}{a}\right)\right), \quad \lambda, \mu, A, B, a \in \mathbf{R},
$$

which satisfies $\varphi_{, 1,2}=-\left(\left(\lambda^{2}+\mu^{2}\right) / a^{2}\right) \varphi$. Considering (5.60) as the curvature $\mathcal{\kappa}$ of a family of curves in the Tchebyshev net, we can construct a surface of constant curvature $K$ by means of the Frenet frame $\{t, n, b\}$ of parametric curves. Frenet formulas are $t^{\prime}=\kappa n, n^{\prime}=\tau b, b^{\prime}=\tau n$, where $\tau= \pm \sqrt{\left(\lambda^{2}+\mu^{2}\right) / a^{2}}$, and they allow us to construct fields $t=\mathbf{x}_{1}$ or $t=\mathbf{x}_{2}$ and $n, b$ and therefore a parametrization $\mathbf{x}=\mathbf{x}(u, v)$. The obtained family of surfaces contains family of surfaces of revolution ( $\lambda=0$ for surfaces obtained by pseudo-Euclidean rotations and $\mu=0$ for surfaces obtained by isotropic rotations). 


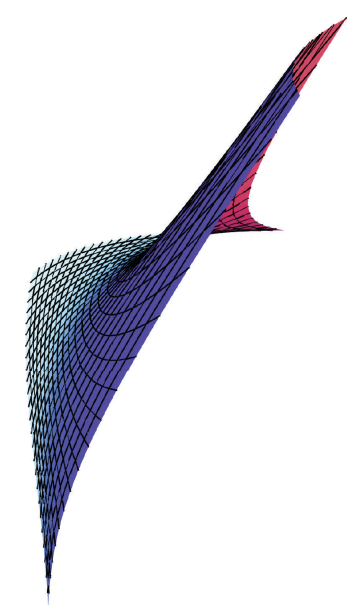

Figure 9: A spacelike surface of constant curvature $K=-5$ with $\lambda=2, \mu=A=a=1, B=0$.

Example 5.9. A surface with parametrization

$$
\begin{aligned}
& x(u, v)=u+v \\
& y(u, v)=\frac{1}{10} e^{2(u-v)}(2 \cos (u+v)+\sin (u+v)(\sqrt{5} \cosh (\sqrt{5}(u-v)-2 \sinh (\sqrt{5}(u-v))))) \\
& z(u, v)=\frac{1}{10} e^{2(u-v)}(2 \cos (u+v)+\sin (u+v)(-2 \cosh (\sqrt{5}(u-v))+\sqrt{5} \sinh \sqrt{5}(u-v)))
\end{aligned}
$$

is a spacelike surface parametrized by the Tchebyshev net having $K=-5$ (see Figure 9).

\section{References}

[1] O. Giering, Vorlesungen über höhere Geometrie, Friedr. Vieweg \& Sohn, Braunschweig, Germany, 1982.

[2] B. Divjak, Geometrija pseudogalilejevih prostora [Ph.D. thesis], University of Zagreb, 1997.

[3] B. Divjak, "Curves in pseudo-Galilean geometry," Annales Universitatis Scientiarum Budapestinensis de Rolando Eötvös Nominatae, vol. 41, pp. 117-128, 1998.

[4] B. Divjak and Ž. Milin-Šipuš, "Special curves on ruled surfaces in Galilean and pseudo-Galilean spaces," Acta Mathematica Hungarica, vol. 98, no. 3, pp. 203-215, 2003.

[5] B. Divjak and Ž. Milin Šipuš, "Minding isometries of ruled surfaces in pseudo-Galilean space," Journal of Geometry, vol. 77, no. 1-2, pp. 35-47, 2003.

[6] B. Divjak and Ž. Milin Šipuš, "Transversal surfaces of ruled surfaces in the pseudo-Galilean space," Sitzungsberichte. Abteilung II, vol. 213, pp. 23-32, 2004.

[7] B. Divjak and Ž. Milin Šipuš, "Some special surfaces in the pseudo-Galilean space," Acta Mathematica Hungarica, vol. 118, no. 3, pp. 209-226, 2008.

[8] Ž. Milin Šipuš, "Ruled Weingarten surfaces in the Galilean space," Periodica Mathematica Hungarica, Journal of the János Bolyai Mathematical Society, vol. 56, no. 2, pp. 213-225, 2008.

[9] Ž. Milin Šipuš and B. Divjak, "Translation surfaces in the Galilean space," Glasnik Matematički. Serija III, vol. 46, no. 2, pp. 455-469, 2011.

[10] O. Röschel, Die Geometrie des Galileischen Raumes, Habilitationsschrift, Leoben, Austria, 1984. 
[11] O. Röschel, "Torusflächen des galileischen Raumes," Studia Scientiarum Mathematicarum Hungarica, vol. 23, no. 3-4, pp. 401-410, 1988.

[12] W. Kühnel, Differential Geometry, Curves_Surfaces-Manifolds, vol. 16 of Student Mathematical Library, American Mathematical Society, Providence, RI, USA, 2002.

[13] N. E. Maryukova, "Surfaces of constant negative curvature in a Galilei space and the Klein-Gordon equation," Rossiǔskaya Akademiya Nauk, vol. 50, no. 1, pp. 203-204, 1995.

[14] B. A. Rosenfeld and N. E. Maryukova, "Surfaces of constant curvature and geometric interpretations of the Klein-Gordon, sine-Gordon and sinh-Gordon equations," Institut Mathématique Publications, vol. 61, pp. 119-132, 1997.

[15] E. Molnár, "The projective interpretation of the eight $G_{3}$-dimensional homogeneous geometries," Beiträge zur Algebra und Geometrie, vol. 38, no. 2, pp. 261-288, 1997.

[16] D. J. Struik, Lectures on Classical Differential Geometry, Dover Publications Inc, New York, NY, USA, 2nd edition, 1988.

[17] S. S. Chern, "Geometrical interpretation of the sinh-Gordon equation," Polska Akademia Nauk. Annales Polonici Mathematici, vol. 39, pp. 63-69, 1981.

[18] H. Sachs, Isotrope Geometrie des Raumes, Friedr. Vieweg \& Sohn, Braunschweig, 1990. 


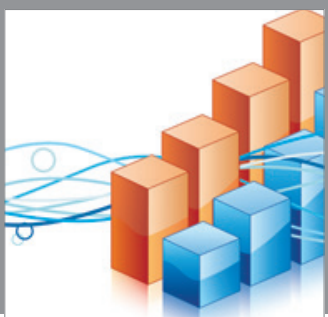

Advances in

Operations Research

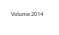

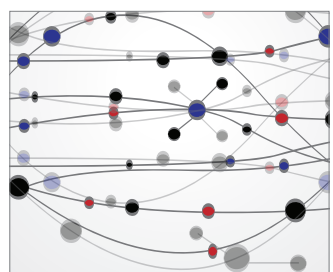

\section{The Scientific} World Journal
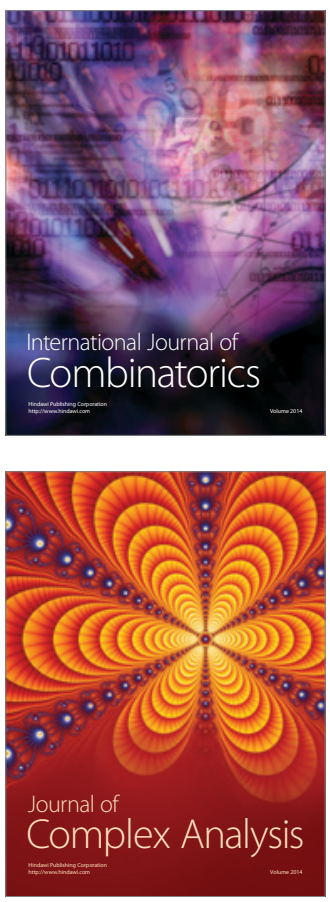

International Journal of

Mathematics and

Mathematical

Sciences
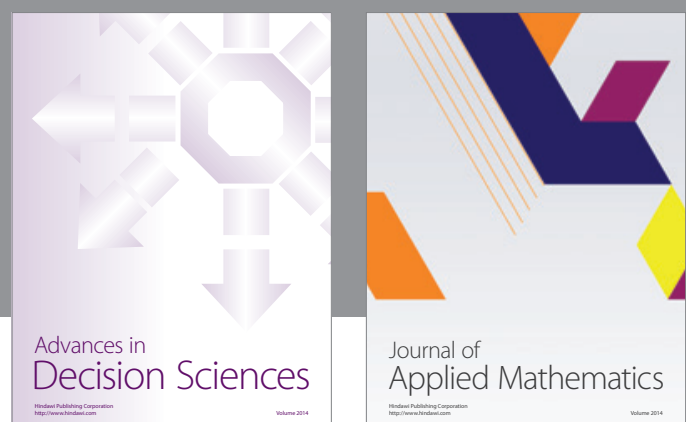

Journal of

Applied Mathematics
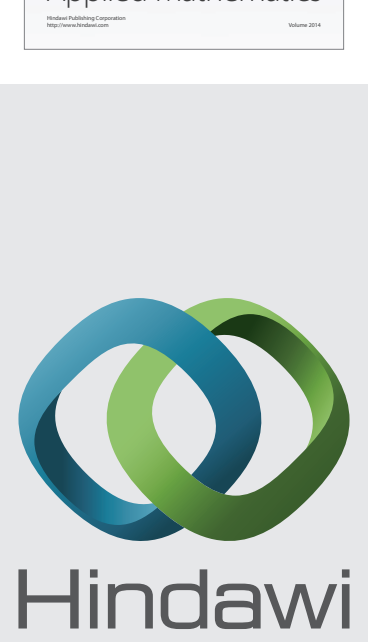

Submit your manuscripts at http://www.hindawi.com
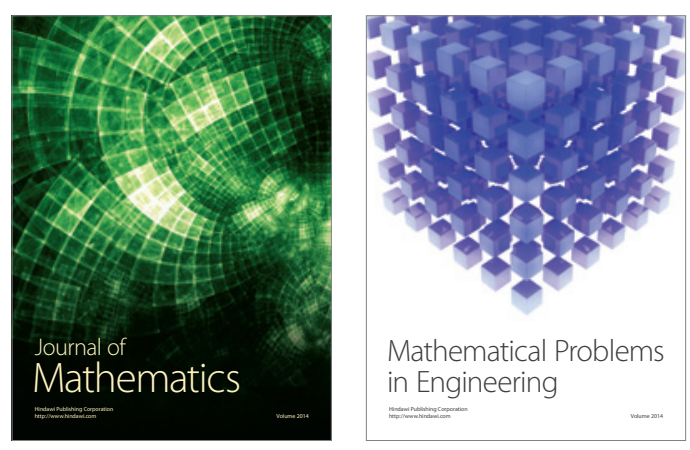

Mathematical Problems in Engineering
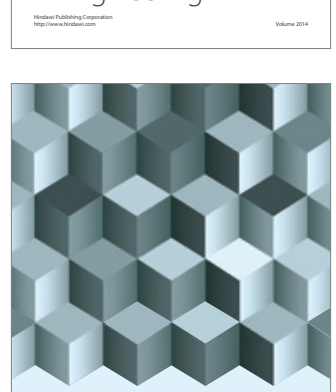

Journal of

Function Spaces
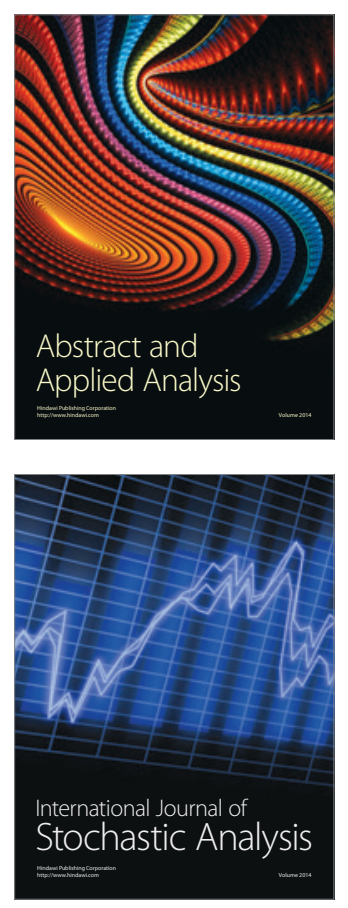

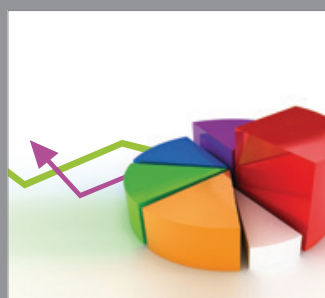

ournal of

Probability and Statistics

Promensencen
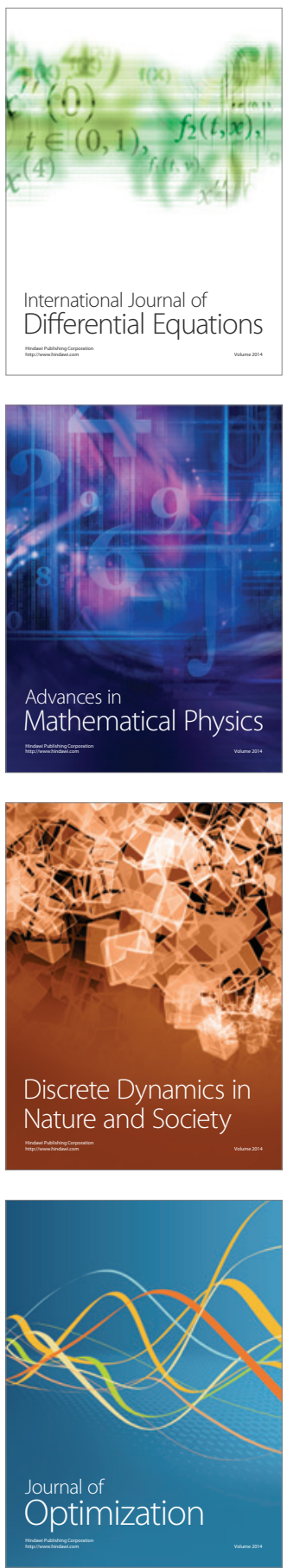